\title{
Bounds for Right Tails of Deterministic and Stochastic Sums of Random Variables
}

\author{
Grzegorz Darkiewicz* Griselda Deelstra ${ }^{\dagger}$

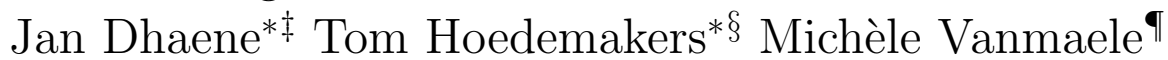

\begin{abstract}
We investigate lower and upper bounds for right tails (stop-loss premiums) of deterministic and stochastic sums of non-independent random variables. The bounds are derived using the concepts of comonotonicity, convex order and conditioning. The performance of the presented approximations is investigated numerically for individual life annuity contracts as well as for life annuity portfolios, where mortality is modeled by Makeham's law, whereas investment returns are modeled by a Brownian motion process.
\end{abstract}

Keywords: stop-loss premium, right tail, comonotonicity, convex order, conditioning, upper and lower bounds.

\section{Introduction}

An insurance risk is described by a random variable (r.v.) $X$ which represents the (discounted) value of future claims of an individual insurance contract or the aggregate claims of an insurance portfolio over a given reference period. One of the main tasks of the actuary is to assess the 'dangerousness' of insurance risks, either by determining their distribution functions or by summarizing their characteristics quantitatively by means of one or more risk measures.

*Department of Applied Economics, Katholieke Universiteit Leuven, Naamsestraat 69, 3000 Leuven, Belgium

${ }^{\dagger}$ Department of Mathematics, ECARES and Solvay Business School, Université Libre de Bruxelles, Boulevard du Triomphe 2, CP 210, 1050 Brussels, Belgium

${ }^{\ddagger}$ Corresponding author. Email address: Jan.Dhaene@econ.kuleuven.be

$\S$ University Center of Statistics, W. de Croylaan 54, 3001 Heverlee, Belgium

"Department of Applied Mathematics and Computer Science, Ghent University, Krijgslaan 281, S9, 9000 Gent, Belgium 
An important class of risk measures related to an insurance risk $X$ consists of the expectations $\mathrm{E}\left[(X-d)_{+}\right]=\mathrm{E}[\max (0, X-d)]$ for different values of $d$. In a reinsurance context, $(X-d)_{+}$can be interpreted as the liability of the reinsurer in a stop-loss reinsurance contract and $\mathrm{E}\left[(X-d)_{+}\right]$is the associated net reinsurance premium, called the stop-loss premium at retention $d$. More generally, the quantity $\mathrm{E}\left[(X-d)_{+}\right]$can be interpreted as a measure for the right tail of the distribution function (d.f.) of $X$, beyond outcome $d$. Intuitively, a r.v. with larger right tails has more probability mass concentrated in the right tail of the d.f. and hence, is 'more dangerous'. In the sequel, we will often call the stop-loss premium $\mathrm{E}\left[(X-d)_{+}\right]$the right tail at level $d$ of (the d.f. of) $X$.

As applications we consider the problem of measuring the right tail of a single life annuity (cash flows with a stochastic time horizon) and of a diversified portfolio of life annuities (cash flows with a deterministic time horizon). Using our results for compound sums we obtain very precise bounds. We provide a number of numerical illustrations which reveal a significant improvement compared with the bounds obtained by traditional comonotonic approximations.

A r.v. $Y$ which has uniformly larger right tails than $X$ is said to be 'larger in the increasing convex order sense', notation $X \leq_{i c x} Y$. In an actuarial context, the increasing convex order is also called stop-loss order, see e.g. Denuit et al. (2005) and the references therein. In terms of utility theory, $X \leq_{i c x} Y$ means that any risk averse decision maker prefers risk $X$ over risk $Y$. Hence, the calculation of right tails of insurance risks makes sense in order to evaluate the 'dangerousness' of the risks at hand. In case $X \leq_{i c x} Y$ and in addition $\mathrm{E}[X]=\mathrm{E}[Y]$, one says that $X$ is smaller than $Y$ in the convex order sense, notation $X \leq_{c x} Y$.

In practice, it is not always straightforward to compute right tails or stop-loss premiums. In the actuarial literature a lot of attention has been devoted to determine bounds for stop-loss premiums in case only partial information about the distribution is available, see e.g. De Vylder and Goovaerts (1982), Jansen et al. (1986), Hürlimann (1996, 1998, 2002).

A particular type of problems arises when determining right tails of a sum $\mathbb{S}=X_{1}+\cdots+X_{n}$ when full information about the distributions of the $X_{i}$ is available but the dependence structure between these $X_{i}$ is not known or too cumbersome to work with. In Dhaene et al. $(2002 \mathrm{a}, \mathrm{b})$ it is shown that in this context the least upper bound of $\mathbb{S}$ in the increasing convex order sense is obtained by replacing the unknown copula of the random vector $\left(X_{1}, X_{2}, \cdots, X_{n}\right)$ by the comonotonic copula. These authors also propose comonotonic lower bounds for the d.f. of $\mathbb{S}$ obtained by conditioning. Such an approach allows to determine analytical bounds for right tails or stop-loss premiums, in particular in the multivariate lognormal case.

In practical applications, the comonotonic lower bounds have proved to be very accurate approximations in case the distributions of the random sum is light to moderate heavy tailed, see e.g. Vanduffel et al. (2005). In Laeven (2005) asymptotic results are derived for the tail probabilities of $\mathbb{S}$ in the presence of heavy-tailedness conditions. 
As opposed to the comonotonic lower bounds, the comonotonic upper bound is only accurate in case of a very strong dependence between summands. Therefore, in this contribution we present a number of techniques which allow to determine improved upper bounds for right tails. Following the ideas presented in Deelstra et al. (2004) and Vanmaele et al. (2006), we use the method of conditioning as in Curran (1994) and in Rogers and Shi (1995). We compare these bounds with the upper and lower bounds for right tails of sums of dependent r.v.'s as derived in Dhaene et al. (2002a,b).

In this paper we are also especially interested in bounds for right tails of a stochastic sum of r.v.'s, which show up in a natural way both in life- and non-life insurances. These bounds follow from the bounds for deterministic sums of r.v.'s, taking into account the tower property for conditional expectations.

In particular, we illustrate our theoretical results by calculating lower and upper bounds for the right tails of the stochastic sum of r.v.'s representing the stochastic present value of future liabilities related with a single life annuity. As a second illustration, we determine lower and upper bounds for the right tails of the deterministic sum of r.v.'s representing the stochastic present value of the future liabilities of a well-diversified portfolio of life annuities.

As the derivation of bounds for a stochastic sum of r.v.'s is based on the study of deterministic sums of r.v.'s, we present different bounds for the latter and do this in the particular situation of sums of lognormal distributed r.v.'s. Such sums are widely encountered in practice, both in actuarial science and in finance. Typical examples are stochastic present values of future cash-flows (see e.g. Dhaene et al. (2002b, 2005)), Asian options (see e.g. Simon et al. (2000), Vanmaele et al. (2006) and Reynaerts et al. (2006)), basket options (see Deelstra et al. (2004) and Vanmaele et al. (2004)) and Asian basket options (see Deelstra et al. (2008)).

Using these bounds, we then propose different bounds for right tails of stochastic sums of dependent r.v.'s in the setting of lognormal distributions, and in particular for our two examples mentioned above, namely a single life annuity (cash flows with a stochastic time horizon) and a diversified portfolio of life annuities (cash flows with a deterministic time horizon). By several numerical experiments, the different bounds are evaluated and compared.

The importance of the proposed bounds lies in the fact that they are analytical and that they are bounds and not only approximations. Monte-Carlo simulations are very timeconsuming compared to the computation of the analytical expressions of these bounds. Multivariate integration on the other hand would require the knowledge of the dependence structure of the involved multivariate random variable which is however not known. The proposed comonotonicity approach circumvents this problem.

The paper is organized as follows. In Section 2 we describe the present value of r.v.'s related to (portfolios of) life annuities, present some basic notations and a simple but important theorem for right tails of a stochastic sum of r.v.'s. In Section 3 we recapitulate the lower and upper bounds of Kaas et al. (2000) and Dhaene et al. (2002a), as well as 
the results of Rogers and Shi (1995). Section 4 explains how the upper bounds can be improved by decomposing right tails. In Section 5 we discuss right tails of compound sums. All presented bounds are illustrated by considering the particular case where all non-independent components constituting the sum are lognormally distributed. In Section 6 we calculate the presented bounds for the life annuity problems described above. Finally, Section 7 concludes the paper.

\section{Life annuities and right tails}

A life annuity underwritten on a life $(x)$ of age $x$ provides a series of periodic payments, where each payment is due conditional on the survival of $(x)$ at the moment of this payment. We denote the future lifetime of $(x)$ by $T_{x}$. Thus $x+T_{x}$ is the age-at-death of the insured. The future lifetime $T_{x}$ is a r.v. with d.f. denoted by

$$
\operatorname{Pr}\left[T_{x} \leq t\right] \stackrel{\text { not. }}{=}{ }_{t} q_{x}=1-{ }_{t} p_{x}, \quad t \geq 0 .
$$

We assume that the d.f. of $T_{x}$ is given. We define $K_{x}=\left\lfloor T_{x}\right\rfloor$, the number of completed future years lived by $(x)$, or the curtate future lifetime of $(x)$, where $\lfloor x\rfloor$ is the largest integer less than or equal to $x$. The d.f. of the integer-valued r.v. $K_{x}$ is given by

$$
\operatorname{Pr}\left[K_{x}=k\right]=\operatorname{Pr}\left[k \leq T_{x}<k+1\right]={ }_{k+1} q_{x}-{ }_{k} q_{x} \stackrel{\text { not. }}{=}{ }_{k \mid} q_{x}, \quad k=0,1, \ldots
$$

In our numerical illustrations, we will assume that the distribution of the remaining lifetime belongs to the Gompertz-Makeham family. Hence, the probabilities defined above follow from a lifetable of the form

$$
l_{x}=a s^{x} g^{c^{x}}, \quad x>0,
$$

with parameters $a>0,0<s<1,0<g<1$ and $c>1^{1}$. See Bowers et al. (1986) for more details.

Consider a whole life annuity on a life $(x)$ which pays an amount of 1 at the end of each year, provided the insured is still alive at that time. Assume that the discounting is performed with a random return. The stochastic present value of the future payments of this annuity is denoted by $\mathbb{S}^{\text {policy }}$ :

$$
\mathbb{S}^{\text {policy }}=\sum_{i=1}^{K_{x}} e^{-Y(i)} .
$$

Here, the r.v.'s $Y(i)$ are defined by $Y(i)=Y_{1}+Y_{2}+\cdots+Y_{i}$, where $Y_{i}$ is the random

\footnotetext{
${ }^{1}$ In our numerical illustrations, we will use the Belgian analytic life table MR for life annuity valuation, with corresponding parameters $l_{0}=1000000 ; a=1000266.63 ; s=0.999441703848 ; g=0.999733441115$ and $c=1.101077536030$.
} 
logreturn over the period $[i-1, i]$ and $e^{-Y(i)}$ is the random discount factor over the period $[0, i]$. We assume that the curtate lifetime $K_{x}$ is independent of the return variables $Y_{i}$.

The r.v. defined in (2) is a special case of a compound sum of the form

$$
\mathbb{S}_{N}=\sum_{i=1}^{N} X_{i}
$$

with $N$ a stochastic number which is independent of the $X_{i}$. The right tails of $\mathbb{S}_{N}$ will be denoted by $\pi\left(\mathbb{S}_{N}, d\right)$. They can be determined using the next result, which follows immediately from the tower property for conditional expectations.

Theorem 1 The right tails $\pi\left(\mathbb{S}_{N}, d\right)=\mathrm{E}\left[\left(\mathbb{S}_{N}-d\right)_{+}\right]$of the compound sum $\mathbb{S}_{N}$ are given by

$$
\pi\left(\mathbb{S}_{N}, d\right)=\sum_{j=1}^{\infty} \operatorname{Pr}(N=j) \pi\left(\mathbb{S}_{j}, d\right), \quad \text { with } \quad \mathbb{S}_{j}=\sum_{i=1}^{j} X_{i}
$$

For the compound sum $\mathbb{S}^{\text {policy }}$ defined in (2), we find from Theorem 1 that

$$
\mathrm{E}\left[\left(\mathbb{S}^{\text {policy }}-d\right)_{+}\right]=\sum_{j=1}^{\infty}{ }_{j} q_{x} \mathrm{E}\left[\left(\tilde{\mathbb{S}}_{j}-d\right)_{+}\right], \quad \text { with } \quad \tilde{\mathbb{S}}_{j}=\sum_{i=1}^{j} e^{-Y(i)}
$$

In a second application we will consider a portfolio of $l_{x}$ life annuity contracts with respective future lifetimes of the insureds $T_{x}^{(1)}, T_{x}^{(2)}, \ldots, T_{x}^{\left(l_{x}\right)}$ which are assumed to be mutually independent. The insurer of this portfolio faces two types of risk: mortality risk and investment risk. The stochastic present value of the portfolio liabilities are given by $\sum_{i=1}^{\infty} L_{x+i} e^{-Y(i)}$, where $L_{x+i}$ denotes the random number of survivors at age $x+i$. The present value of the liabilities per portfolio are then given by $\sum_{i=1}^{\infty} \frac{L_{x+i}}{l_{x}} e^{-Y(i)}$. From the Law of Large Numbers it follows that the mortality risk decreases with the number of policies $l_{x}$ while the investment risk remains the same as each of the policies is exposed to the same investment risk. Thus for a sufficiently large number of policies the present value of the liabilities per policy can be approximated by

$$
\mathbb{S}^{\text {average }}=\sum_{i=1}^{\infty}{ }_{i} p_{x} e^{-Y(i)}
$$

The right tails of the present value of the portfolio liabilities per policy can then be approximated by

$$
\mathrm{E}\left[\left(\sum_{i=1}^{\infty} \frac{L_{x+i}}{l_{x}} e^{-Y(i)}-d\right)_{+}\right] \approx \mathrm{E}\left[\left(\sum_{i=1}^{\infty}{ }_{i} p_{x} e^{-Y(i)}-d\right)_{+}\right] .
$$


Note that in practice there exists a $t$ such that ${ }_{t} p_{x}=0$ for all $t \geq 0$. In this case, the infinite sums that appear in our previous derivations will all transform in a finite sum and the r.v.'s $\widetilde{\mathbb{S}}_{j}$ in (5) and $\mathbb{S}^{\text {average }}$ in (6) are both of the form

$$
\mathbb{S}=\sum_{i=1}^{n} X_{i}=\sum_{i=1}^{n} \alpha_{i} e^{Z_{i}}
$$

where $Z_{i} \sim N\left(\mathrm{E}\left[Z_{i}\right], \sigma_{Z_{i}}\right)$ and $\alpha_{i} \geq 0$.

\section{Bounds based on comonotonicity and conditioning}

\subsection{Lower and upper bounds for the d.f. of $\mathbb{S}$}

In the life annuity applications mentioned in the previous section, we are confronted with a r.v. of the type $\mathbb{S}=\sum_{i=1}^{n} X_{i}$ as defined in (7) where the d.f.'s of the terms $X_{i}$ are given, but the multivariate d.f. of the random vector $\left(X_{1}, X_{2}, \ldots, X_{n}\right)$ is not completely specified or too cumbersome to work with. In such cases it may be helpful to find r.v.'s $\mathbb{S}_{1}$ and $\mathbb{S}_{2}$ such that

$$
\mathbb{S}_{1} \leq_{c x} \mathbb{S} \leq_{c x} \mathbb{S}_{2}
$$

which implies by definition of convex order that the corresponding right tails satisfy

$$
\mathrm{E}\left[\left(\mathbb{S}_{1}-d\right)_{+}\right] \leq \mathrm{E}\left[(\mathbb{S}-d)_{+}\right] \leq \mathrm{E}\left[\left(\mathbb{S}_{2}-d\right)_{+}\right]
$$

for all $d$.

Let us first consider the case that the only information that is available concerning the multivariate d.f. of the random vector $\left(X_{1}, \ldots, X_{n}\right)$ is the marginal d.f.'s of the $X_{i}$. Let $U$ denote a uniformly distributed r.v. on the unit interval $(0,1)$ and let $F_{X}^{-1}$ be the inverse of the d.f. of $X$ defined as usual as

$$
F_{X}^{-1}(p)=\inf \left\{x \in \mathbb{R} \mid F_{X}(x) \geq p\right\}, \quad p \in[0,1],
$$

with $\inf \emptyset=+\infty$ by convention. In this case the d.f. of the comonotonic sum

$$
\mathbb{S}^{c u b}=F_{X_{1}}^{-1}(U)+F_{X_{2}}^{-1}(U)+\cdots+F_{X_{n}}^{-1}(U),
$$

is a prudent choice for approximating the unknown d.f. of $\mathbb{S}$ because $\mathbb{S} \leq_{c x} \mathbb{S}^{c u b}$. It is a supremum in terms of convex order in the sense that in the class of all random sums with given marginals any stop-loss premium of the comonotonic sum dominates the corresponding stop-loss premiums of all other sums. In the sequel, we denote the right tails or stop-loss premiums of $\mathbb{S}^{c u b}$ by $\pi^{c u b}(\mathbb{S}, d)$.

From Denneberg (1994) and Landsberger et al. (1994), it is known that the inverse distribution function of a sum of comonotonic r.v.'s is simply the sum of the inverse distribution 
functions of the marginal distributions. Then it follows that the stop-loss premiums of a sum of comonotonic r.v.'s can be easily obtained as a linear combination of the appropriate stop-loss premiums of the terms, see Dhaene et al. (2002a). In the case of strictly increasing marginals, the right tails $\pi^{c u b}(\mathbb{S}, d)$ can be expressed as

$$
\pi^{c u b}(\mathbb{S}, d)=\sum_{i=1}^{n} \pi\left(X_{i}, F_{X_{i}}^{-1}\left(F_{\mathbb{S}}(d)\right)\right), \quad F_{\mathbb{S}^{c}}^{-1}(0)<d<F_{\mathbb{S}^{c}}^{-1}(1),
$$

with the d.f. $F_{\mathbb{S}^{c}}(d)$ implicitly determined by

$$
\sum_{i=1}^{n} F_{X_{i}}^{-1}\left(F_{\mathbb{S}^{c}}(d)\right)=d, \quad F_{\mathbb{S}^{c}}^{-1}(0)<d<F_{\mathbb{S}^{c}}^{-1}(1) .
$$

For strictly increasing and continuous marginals $F_{\mathbb{S}^{c}}(d)$ is uniquely determined by (8). Applying this result to the sum $\mathbb{S}$ of lognormal r.v.'s as defined in (7), we find the following expression for $\pi^{c u b}(\mathbb{S}, d)$ :

$$
\pi^{c u b}(\mathbb{S}, d)=\sum_{i=1}^{n} \alpha_{i} e^{\mathrm{E}\left[Z_{i}\right]+\frac{\sigma_{Z_{i}}^{2}}{2}} \Phi\left[\sigma_{Z_{i}}-\Phi^{-1}\left(F_{\mathbb{S}^{c}}(d)\right)\right]-d\left(1-F_{\mathbb{S}^{c}}(d)\right),
$$

where $F_{\mathbb{S}^{c}}(d)$ can be found by solving

$$
\sum_{i=1}^{n} \alpha_{i} e^{\mathrm{E}\left[Z_{i}\right]+\sigma_{Z_{i}} \Phi^{-1}\left(F_{\mathbb{S} c}(d)\right)}=d, \quad F_{\mathbb{S}^{c}}^{-1}(0)<d<F_{\mathbb{S}^{c}}^{-1}(1),
$$

see Dhaene et al. (2002b) for more details.

We can improve the comonotonic upper bound $\pi^{c u b}(\mathbb{S}, d)$ when there is some additional information available concerning the stochastic nature of $\left(X_{1}, \ldots, X_{n}\right)$. More precisely, we assume that there exists a r.v. $\Lambda$ with a given d.f. and such that we know the conditional d.f.'s of the r.v. $X_{i}$, given $\Lambda=\lambda$, for all possible values of $\lambda$. Kaas et al. (2000) define the improved comonotonic upper bound $\mathbb{S}^{u}$ as

$$
\mathbb{S}^{u}=F_{X_{1} \mid \Lambda}^{-1}(U)+F_{X_{2} \mid \Lambda}^{-1}(U)+\cdots+F_{X_{n} \mid \Lambda}^{-1}(U),
$$

where $F_{X_{i} \mid \Lambda}^{-1}(U)$ is the notation for the r.v. $f_{i}(U, \Lambda)$, with the function $f_{i}$ defined by $f_{i}(u, \lambda)=F_{X_{i} \mid \Lambda=\lambda}^{-1}(u)$.

An expression for the right tails $\mathrm{E}\left[\left(\mathbb{S}^{u}-d\right)_{+}\right]$, further denoted by $\pi^{i c u b}(\mathbb{S}, d, \Lambda)$, of the improved comonotonic upper bound $\mathbb{S}^{u}$ for $\mathbb{S}$ can be found in Kaas et al. (2000).

Let us now consider the sum of lognormal r.v.'s $\mathbb{S}$ defined in (7) and a conditioning variable $\Lambda$ satisfying the following assumption:

Assumption 1: $\Lambda$ is a normally distributed r.v. for which the $\left(Z_{i}, \Lambda\right), i=1, \ldots, n$, are bivariate normally distributed. 
Under this assumption, we find from Kaas et al. (2000) that

$$
\begin{aligned}
\pi^{i c u b}(\mathbb{S}, d, \Lambda)= & \sum_{i=1}^{n} \alpha_{i} e^{\mathrm{E}\left[Z_{i}\right]+\frac{1}{2} \sigma_{Z_{i}}^{2}\left(1-r_{i}^{2}\right)} \int_{0}^{1} e^{r_{i} \sigma_{Z_{i}} \Phi^{-1}(v)} \times \\
& \times \Phi\left(\sqrt{1-r_{i}^{2}} \sigma_{Z_{i}}-\Phi^{-1}\left(F_{\mathbb{S}^{u}}(d \mid V=v)\right)\right) d v-d\left(1-F_{\mathbb{S}^{u}}(d)\right)
\end{aligned}
$$

where the correlations $r_{i}, i=1, \ldots, n$, are defined by

$$
r_{i}=\operatorname{Corr}\left(Z_{i}, \Lambda\right)=\frac{\operatorname{Cov}\left[Z_{i}, \Lambda\right]}{\sigma_{Z_{i}} \sigma_{\Lambda}},
$$

and where $V=\Phi\left(\frac{\Lambda-\mathrm{E}[\Lambda]}{\sigma_{\Lambda}}\right)$. The conditional probability $F_{\mathbb{S}^{u}}(d \mid V=v)$, is the d.f. of a sum of $n$ comonotonic r.v.'s and follows, for $F_{\mathbb{S}^{u} \mid V=v}^{-1}(0)<d<F_{\mathbb{S}^{u} \mid V=v}^{-1}(1)$, implicitly from:

$$
\sum_{i=1}^{n} \alpha_{i} e^{\mathrm{E}\left[Z_{i}\right]+r_{i} \sigma_{Z_{i}} \Phi^{-1}(v)+\sqrt{1-r_{i}^{2}} \sigma_{Z_{i}} \Phi^{-1}\left(F_{\mathbb{S}^{u}}(d \mid V=v)\right)}=d .
$$

Remark that when all $r_{i}$ are zero the improved comonotonic upper bound coincides with the comonotonic upper bound. On the other hand, when the retention becomes very large both comonotonic bounds will converge to one another and tend in the limit to zero.

The additional information described via a r.v. $\Lambda$, as mentioned above, can also be used to construct the convex order lower bound $\mathbb{S}^{\ell}=\mathrm{E}[\mathbb{S} \mid \Lambda]$ for $\mathbb{S}$, see e.g. Rogers and Shi (1995) and Kaas et al. (2000). However in contrast to the upper bound $\mathbb{S}^{u}$ which is a comonotonic sum by construction, we now have to make additional assumptions on $\Lambda$ to guarantee that this lower bound is a comonotonic sum.

When the r.v. $\Lambda$ is such that all $\mathrm{E}\left[X_{i} \mid \Lambda\right]$ are non-decreasing (or all are non-increasing) and continuous functions of $\Lambda$, then $\mathbb{S}^{\ell}$ is a comonotonic sum, see Kaas et al. (2000). Let us assume that the d.f.'s of the r.v.'s $\mathrm{E}\left[X_{i} \mid \Lambda\right]$ are strictly increasing. In this case the right tails $\mathrm{E}\left[\left(\mathbb{S}^{\ell}-d\right)_{+}\right]$, denoted by $\pi^{\ell b}(\mathbb{S}, d, \Lambda)$, of the lower bound $\mathbb{S}^{\ell}$ for $\mathbb{S}$ are given by

$$
\pi^{\ell b}(\mathbb{S}, d, \Lambda)=\sum_{i=1}^{n} \pi\left(\mathrm{E}\left[X_{i} \mid \Lambda\right], F_{\mathrm{E}\left[X_{i} \mid \Lambda\right]}^{-1}\left(F_{\mathbb{S}^{\ell}}(d)\right)\right), \quad F_{\mathbb{S} \ell}^{-1}(0)<d<F_{\mathbb{S} \ell}^{-1}(1),
$$

with $\Lambda$ the conditioning r.v. in the definition of $\mathbb{S}^{\ell}$ and where $F_{\mathbb{S}^{\ell}}(d)$ can be obtained from

$$
\sum_{i=1}^{n} F_{\mathrm{E}\left[X_{i} \mid \Lambda\right]}^{-1}\left(F_{\mathbb{S} \ell}(d)\right)=d
$$

This last equation has a unique solution when the d.f.'s of the r.v.'s $\mathrm{E}\left[X_{i} \mid \Lambda\right]$ are strictly increasing and continuous. 
Remark 1 In case the cumulative d.f.'s of the r.v.'s $\mathrm{E}\left[X_{i} \mid \Lambda\right]$ are not continuous nor strictly increasing or decreasing functions of $\Lambda$, then the stop-loss premiums of $\mathbb{S}^{\ell}$, which is not comonotonic anymore, can be determined as follows:

$$
\pi^{\ell b}(\mathbb{S}, d, \Lambda)=\int_{-\infty}^{+\infty}\left(\sum_{i=1}^{n} \mathrm{E}\left[X_{i} \mid \Lambda=\lambda\right]-d\right)_{+} d F_{\Lambda}(\lambda) .
$$

Let us now again assume that $\mathbb{S}$ is a sum of lognormal r.v.'s as defined in (7) and that Assumption 1 holds. Furthermore, we assume that the following assumption holds:

Assumption 2: All correlations $r_{i}$ in (10) are non-negative, i.e. $r_{i} \geq 0$ for all $i$.

The case $r_{i} \leq 0$ for all $i$ can be treated in a similar way.

For the right tail $\pi^{\ell b}(\mathbb{S}, d, \Lambda)$ of $\mathbb{S}^{\ell}$, which is a lower bound for $\pi(\mathbb{S}, d)$, we find the following expression for (12), see Dhaene et al. (2002b):

$$
\pi^{\ell b}(\mathbb{S}, d, \Lambda)=\sum_{i=1}^{n} \alpha_{i} e^{\mathrm{E}\left[Z_{i}\right]+\frac{1}{2} \sigma_{Z_{i}}^{2}} \Phi\left[r_{i} \sigma_{Z_{i}}-\Phi^{-1}\left(F_{\mathbb{S}^{\ell}}(d)\right)\right]-d\left(1-F_{\mathbb{S}^{\ell}}(d)\right),
$$

where $F_{\mathbb{S} \ell}(d)$ is the unique solution to

$$
\sum_{i=1}^{n} \alpha_{i} e^{\mathrm{E}\left[Z_{i}\right]+r_{i} \sigma_{Z_{i}} \Phi^{-1}\left(F_{\mathbb{S} \ell}(d)\right)+\frac{1}{2}\left(1-r_{i}^{2}\right) \sigma_{Z_{i}}^{2}}=d .
$$

Note that in case of perfect correlation, i.e. all $r_{i}=1$, the comonotonic sums $\mathbb{S}^{\ell}$ and $\mathbb{S}^{c}$, as well as their respective right tails coincide.

\subsection{Upper bound based on lower bound plus error term}

Applying the following general inequality for any r.v. $Y$ and $Z$ from Rogers and Shi (1995):

$$
0 \leq \mathrm{E}\left[\mathrm{E}\left[Y_{+} \mid Z\right]-\mathrm{E}[Y \mid Z]_{+}\right] \leq \frac{1}{2} \mathrm{E}[\sqrt{\operatorname{Var}(Y \mid Z)}]
$$

to the case of $Y$ being $\mathbb{S}-d$ and $Z$ being the conditioning variable $\Lambda$ in the definition of $\mathbb{S}^{\ell}$, we obtain by an analogous reasoning as in Nielsen and Sandmann (2003) and Vanmaele et al. (2006), an upper bound $\pi^{e u b}(\mathbb{S}, d, \Lambda)$ for the stop-loss premium $\pi(\mathbb{S}, d)$ with retention $d$ based on the lower bound:

$$
\pi(\mathbb{S}, d) \leq \pi^{\ell b}(\mathbb{S}, d, \Lambda)+\varepsilon=: \pi^{e u b}(\mathbb{S}, d, \Lambda),
$$


where the error bound $\varepsilon$ equals

$$
\varepsilon:=\frac{1}{2} \mathrm{E}[\sqrt{\operatorname{Var}(Y \mid Z)}]=\frac{1}{2} \mathrm{E}\left[\left(\sum_{i=1}^{n} \sum_{j=1}^{n} \mathrm{E}\left[X_{i} X_{j} \mid \Lambda\right]-\left(\mathbb{S}^{\ell}\right)^{2}\right)^{1 / 2}\right] .
$$

Note that the error bound is independent of the retention $d$. Thus this upper bound (16) is a constant deviation of the lower bound for any value of the retention $d$. This implies that for large retentions for which the stop-loss premiums will be smaller than for small retentions, this upper bound will not perform very well.

Specifying that the components $X_{i}$ in $\mathbb{S}$ are lognormally distributed according to (7) and imposing Assumption 1 on the conditioning r.v. $\Lambda$, we can express the error bound more explicitly :

$$
\begin{aligned}
\varepsilon=\frac{1}{2} \int_{0}^{1}\left\{\sum_{i=1}^{n} \sum_{j=1}^{n} \alpha_{i} \alpha_{j} e^{\mathrm{E}\left[Z_{i j}\right]+r_{i j} \sigma_{Z_{i j}} \Phi^{-1}(v)+\frac{1}{2}\left(1-r_{i j}^{2}\right) \sigma_{Z_{i j}}^{2}}\right. \\
\left.-\left(\sum_{i=1}^{n} \alpha_{i} e^{\mathrm{E}\left[Z_{i}\right]+r_{i} \sigma_{Z_{i}} \Phi^{-1}(v)+\frac{1}{2}\left(1-r_{i}^{2}\right) \sigma_{Z_{i}}^{2}}\right)^{2}\right\}^{\frac{1}{2}} d v
\end{aligned}
$$

where $Z_{i j}=Z_{i}+Z_{j}$ with $\mathrm{E}\left[Z_{i j}\right]=\mathrm{E}\left[Z_{i}\right]+\mathrm{E}\left[Z_{j}\right]$ and $\sigma_{Z_{i j}}^{2}=\sigma_{Z_{i}}^{2}+\sigma_{Z_{j}}^{2}+2 \operatorname{Cov}\left(Z_{i}, Z_{j}\right)$ and where $r_{i j}$ stands for the correlation of this $Z_{i j}$ and $\Lambda$ :

$$
r_{i j}=\frac{\operatorname{Cov}\left(Z_{i j}, \Lambda\right)}{\sigma_{Z_{i j}} \sigma_{\Lambda}}=\frac{\operatorname{Cov}\left(Z_{i}, \Lambda\right)}{\sigma_{Z_{i j}} \sigma_{\Lambda}}+\frac{\operatorname{Cov}\left(Z_{j}, \Lambda\right)}{\sigma_{Z_{i j}} \sigma_{\Lambda}}=\frac{\sigma_{Z_{i}}}{\sigma_{Z_{i j}}} r_{i}+\frac{\sigma_{Z_{j}}}{\sigma_{Z_{i j}}} r_{j} .
$$

Substituting this expression (18) for the error bound and relation (14) for the lower bound into (16) provides an analytical expression for the upper bound $\pi^{e u b}(\mathbb{S}, d, \Lambda)$ in the lognormal case under the Assumptions 1 and 2. This expression is therefore more general than the error obtained in Theorem 5 of Vanmaele et al. (2006), which concentrates upon Asian options in a Black \& Scholes framework.

\section{Bounds by conditioning through decomposition of the right tail}

In this section we show how to improve the upper bounds introduced in the previous section. The idea is to split off that part of the right tail that can be calculated exactly and to bound the remaining part. 


\subsection{Decomposition of the right tail}

We condition $\mathbb{S}$ on some r.v. $\Lambda$ and impose one of the following assumptions:

Assumption 3: There exists a $d_{\Lambda}$ such that $\Lambda \geq d_{\Lambda}$ implies that $\mathbb{S} \geq d$.

Assumption 4: There exists a $d_{\Lambda}$ such that $\Lambda \leq d_{\Lambda}$ implies that $\mathbb{S} \geq d$.

Then the right tail of $\mathbb{S}$ can be decomposed in two parts, one of which can either be computed exactly or by using numerical integration, depending on the distribution of the underlying r.v.. For the remaining part we first derive a lower and an upper bound based on comonotonic risks, and another upper bound equal to that lower bound plus an error term. This decomposition idea goes back at least to Curran (1994).

Theorem 2 Under Assumption 3 the right tail of $\mathbb{S}$ with retention $d$ can be decomposed in $I_{1}$ and $I_{2}$, i.e. $\pi(\mathbb{S}, d)=I_{1}+I_{2}$, with

$$
\begin{aligned}
& I_{1}=\int_{-\infty}^{d_{\Lambda}} \mathrm{E}\left[(\mathbb{S}-d)_{+} \mid \Lambda=\lambda\right] d F_{\Lambda}(\lambda), \\
& I_{2}=\int_{d_{\Lambda}}^{+\infty} \sum_{i=1}^{n} \mathrm{E}\left[X_{i} \mid \Lambda=\lambda\right] d F_{\Lambda}(\lambda)-d\left(1-F_{\Lambda}\left(d_{\Lambda}\right)\right) .
\end{aligned}
$$

This result has been derived and used in the proof of Theorem 7 of Vanmaele et al. (2006).

Remark 2 1. Under Assumption 4 a similar decomposition holds with the appropriate integration bounds. Also in the following sections, the case of Assumption 4 can be treated in a similar way but will not be mentioned any further.

2. In practical applications the existence of such a $d_{\Lambda}$ depends on the actual form of $\mathbb{S}$ and $\Lambda$.

3. The second integral $I_{2}$ can be written out explicitly if the bivariate distribution of $\left(X_{i}, \Lambda\right)$ is known for all $i$.

\subsection{Lower bound}

By means of Jensen's inequality, the first integral $I_{1}$ in (19) can be bounded below:

$$
I_{1} \geq \int_{-\infty}^{d_{\Lambda}}(\mathrm{E}[\mathbb{S} \mid \Lambda=\lambda]-d)_{+} d F_{\Lambda}(\lambda)=\int_{-\infty}^{d_{\Lambda}}\left(\sum_{i=1}^{n} \mathrm{E}\left[X_{i} \mid \Lambda=\lambda\right]-d\right)_{+} d F_{\Lambda}(\lambda) .
$$

By adding the exact part (20) and using the definition of $\mathbb{S}^{\ell}$, we end up with the lower bound (12). 
Thus, when $\mathbb{S}^{\ell}$ is a sum of $n$ comonotonic risks we can apply (12) which holds even when we do not know or find an appropriate $d_{\Lambda}$.

When $\mathbb{S}^{\ell}$ is not comonotonic we use under Assumption 3 the decomposition

$\pi^{\ell b}(\mathbb{S}, d, \Lambda)=\int_{-\infty}^{d_{\Lambda}}\left(\sum_{i=1}^{n} \mathrm{E}\left[X_{i} \mid \Lambda=\lambda\right]-d\right)_{+} d F_{\Lambda}(\lambda)+\int_{d_{\Lambda}}^{+\infty} \sum_{i=1}^{n} \mathrm{E}\left[X_{i} \mid \Lambda=\lambda\right] d F_{\Lambda}(\lambda)-d\left(1-F_{\Lambda}\left(d_{\Lambda}\right)\right)$,

instead of formula (13).

\subsection{Upper bound based on lower bound}

Under Assumption 3 we improve the bound (16) by making the error bound $\varepsilon(17)$ dependent on the integration bound $d_{\Lambda}$.

Theorem 3 Under Assumption 3 the upper bound $\pi^{\text {deub }}$ for the stop-loss premium $\pi(\mathbb{S}, d)$ with retention $d$ is given by

$$
\pi^{\operatorname{deub}}(\mathbb{S}, d, \Lambda)=\pi^{\ell b}(\mathbb{S}, d, \Lambda)+\varepsilon\left(d_{\Lambda}\right),
$$

where the error bound $\varepsilon\left(d_{\Lambda}\right)$ equals

$$
\varepsilon\left(d_{\Lambda}\right):=\frac{1}{2}\left(\mathrm{E}\left[\operatorname{Var}(\mathbb{S} \mid \Lambda) 1_{\left\{\Lambda<d_{\Lambda}\right\}}\right]\right)^{\frac{1}{2}}\left(\mathrm{E}\left[1_{\left\{\Lambda<d_{\Lambda}\right\}}\right]\right)^{\frac{1}{2}},
$$

where $1_{\left\{\Lambda<d_{\Lambda}\right\}}$ is the indicator function, i.e. $1_{\{c\}}=1$ if the condition $c$ is true and $1_{\{c\}}=0$ if it is not.

This result can be found in the reasoning leading to equation (4) in Nielsen and Sandmann (2003).

Remark 3 As an intermediate step in the derivation of the upper bound (21) we find the following upper bound:

$$
\pi(\mathbb{S}, d) \leq \pi^{\ell b}(\mathbb{S}, d, \Lambda)+\frac{1}{2} \int_{-\infty}^{d_{\Lambda}}(\operatorname{Var}(\mathbb{S} \mid \Lambda=\lambda))^{\frac{1}{2}} d F_{\Lambda}(\lambda)
$$

The limiting case of this upper bound where $d_{\Lambda}$ equals infinity is precisely the upper bound (16) which is hence independent of $d_{\Lambda}$. Applying Hölders inequality to the integral in (23) yields the result (21). Obviously, the error bound in (23) improves the error bound (17). In practical applications, the additional error introduced by Hölders inequality turns out to be much smaller than the difference $\frac{1}{2} \mathrm{E}[\sqrt{\operatorname{Var}(\mathbb{S} \mid \Lambda)}]-\varepsilon\left(d_{\Lambda}\right)$. 
Considering the lognormal case we can write out an explicit expression for the error bound $\varepsilon\left(d_{\Lambda}\right)$ under Assumptions 1 and 3 :

$$
\begin{aligned}
\varepsilon\left(d_{\Lambda}\right)=\frac{1}{2} \Phi\left(d_{\Lambda}^{*}\right)^{1 / 2}\left\{\sum_{i=1}^{n}\right. & \sum_{j=1}^{n} \alpha_{i} \alpha_{j} e^{\mathrm{E}\left[Z_{i}\right]+s E\left[Z_{j}\right]+\frac{1}{2}\left(\sigma_{Z_{i}}^{2}+\sigma_{Z_{j}}^{2}\right)} \\
& \left.\times \Phi\left(d_{\Lambda}^{*}-\left(r_{i} \sigma_{Z_{i}}+r_{j} \sigma_{Z_{j}}\right)\right)\left(e^{\operatorname{Cov}\left(Z_{i}, Z_{j}\right)}-e^{\sigma_{Z_{i}} \sigma_{Z_{j}} r_{i} r_{j}}\right)\right\}^{\frac{1}{2}},
\end{aligned}
$$

with $d_{\Lambda}^{*}=\frac{d_{\Lambda}-\mathrm{E}[\Lambda]}{\sigma_{\Lambda}}$. Combining this expression (24) for the error bound with (14) for the lower bound provides an analytical expression for the upper bound (21) under the additional Assumption 2 for $\Lambda$.

\subsection{Partially exact/comonotonic upper bound}

Under Assumption 3 another upper bound can be obtained as is explained in section 2.3 of Vanmaele et al. (2006) for Asian option pricing in the Black \& Scholes framework. In general, we bound the first term $I_{1}$ in (19) by replacing $\mathbb{S} \mid \Lambda=\lambda$ by its comonotonic upper bound $\mathbb{S}^{u}(9)$ (in convex order sense):

$$
\int_{-\infty}^{d_{\Lambda}} \mathrm{E}\left[(\mathbb{S}-d)_{+} \mid \Lambda=\lambda\right] d F_{\Lambda}(\lambda) \leq \int_{-\infty}^{d_{\Lambda}} \mathrm{E}\left[\left(\mathbb{S}^{u}-d\right)_{+} \mid \Lambda=\lambda\right] d F_{\Lambda}(\lambda) .
$$

Adding (25) to the exact part (20) of the decomposition of the stop-loss premium of $\mathbb{S}$ results in the so-called partially exact/comonotonic upper bound for a stop-loss premium. We will use the notation $\pi^{\text {pecub }}(\mathbb{S}, d, \Lambda)$ to indicate this upper bound.

Theorem 4 Under Assumption 3 the partially exact/comonotonic upper bound for $\pi(\mathbb{S}, d)$ is given by

$$
\begin{aligned}
& \pi^{\text {pecub }}(\mathbb{S}, d, \Lambda) \\
& =\int_{-\infty}^{d_{\Lambda}} E\left[\left(\mathbb{S}^{u}-d\right)_{+} \mid \Lambda=\lambda\right] d F_{\Lambda}(\lambda)+\int_{d_{\Lambda}}^{+\infty} \sum_{i=1}^{n} \mathrm{E}\left[X_{i} \mid \Lambda=\lambda\right] d F_{\Lambda}(\lambda)-d\left(1-F_{\Lambda}\left(d_{\Lambda}\right)\right) .
\end{aligned}
$$

Remark 4 It is easily seen that

$$
\pi^{\text {pecub }}(\mathbb{S}, d, \Lambda) \leq \pi^{i c u b}(\mathbb{S}, d, \Lambda),
$$

while for two distinct conditioning variables $\Lambda_{1}$ and $\Lambda_{2}$ it does not necessarily hold that

$$
\pi^{p e c u b}\left(\mathbb{S}, d, \Lambda_{1}\right) \leq \pi^{i c u b}\left(\mathbb{S}, d, \Lambda_{2}\right)
$$


For the lognormal case we impose again Assumptions 1 and 3. Then the partially exact/comonotonic upper bound is given by

$$
\begin{aligned}
& \pi^{p e c u b}(\mathbb{S}, d, \Lambda) \\
& =\sum_{i=1}^{n} \alpha_{i} e^{\mathrm{E}\left[Z_{i}\right]+\frac{1}{2} \sigma_{Z_{i}}^{2}\left(1-r_{i}^{2}\right)}\left\{\int_{0}^{\Phi\left(d_{\Lambda}^{*}\right)} e^{r_{i} \sigma_{Z_{i}} \Phi^{-1}(v)} \Phi\left(\operatorname{sign}\left(\alpha_{i}\right) \sqrt{1-r_{i}^{2}} \sigma_{Z_{i}}-\Phi^{-1}\left(F_{\mathbb{S} u \mid V=v}(d)\right)\right) d v\right.
\end{aligned}
$$

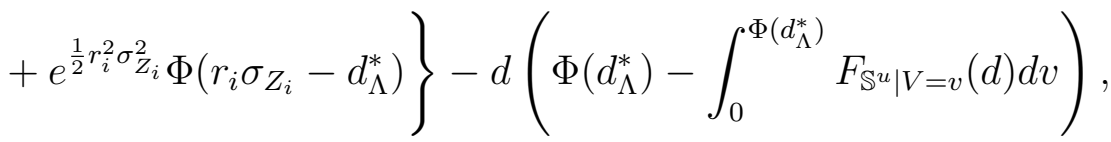

with $d_{\Lambda}^{*}=\frac{d_{\Lambda}-\mathrm{E}[\Lambda]}{\sigma_{\Lambda}}$ and where $F_{\mathbb{S}^{u} \mid V=v}(d)$ can be obtained from (11).

\subsection{Choice of the conditioning r.v. in the multivariate lognormal case}

This subsection is restricted to sums $\mathbb{S}$ of lognormal r.v.'s as presented in (7). The conditioning r.v. $\Lambda$ in the bounds presented above has to be chosen. Obviously, an 'optimal' choice will provide a better bound. Different choices for a r.v. $\Lambda$ of the form

$$
\Lambda=\sum_{i=1}^{n} \gamma_{i} Z_{i}
$$

in the multivariate lognormal case are discussed and compared in detail in Vanduffel et al. (2005).

Kaas, Dhaene and Goovaerts (2000) propose the following choice for the parameters $\gamma_{i}$ when computing the lower bound $\mathbb{S}^{\ell}$ :

$$
\gamma_{i}=\alpha_{i} e^{\mathrm{E}\left[Z_{i}\right]}, \quad i=1, \ldots, n
$$

This 'Taylor-based' choice makes $\Lambda$ a linear transformation of a first order approximation to $\mathbb{S}$.

In view of (27), a possible decomposition variable $d_{\Lambda}$ for which $\Lambda \geq d_{\Lambda}$ implies that $\mathbb{S} \geq d$ is given by

$$
d_{\Lambda}=d-\sum_{i=1}^{n} \gamma_{i}\left(1-\ln \gamma_{i}+\ln \alpha_{i}\right)
$$

Vanduffel et al. (2005) propose to use the conditioning r.v. $\Lambda$ defined in (26) with the coefficients $\gamma_{i}$ given by

$$
\gamma_{i}=\alpha_{i} e^{\mathrm{E}\left[Z_{i}\right]+\frac{1}{2} \sigma_{Z_{i}}^{2}}, \quad i=1, \ldots, n .
$$

This choice can be seen as the one that maximizes a first order approximation of the variance of $\mathbb{S}^{\ell}$. 
For this 'maximal variance' conditioning variable a possible choice for $d_{\Lambda}$ is also of the form (28).

A third possibility for the choice of the conditioning r.v. $\Lambda$ is based on the standardized logarithm of the geometric average $\mathbb{G}=\left(\prod_{i=1}^{n} X_{i}\right)^{1 / n}$ as in Nielsen and Sandmann (2003)

$$
\Lambda=\frac{\ln \mathbb{G}-\mathrm{E}[\ln \mathbb{G}]}{\sqrt{\operatorname{Var}(\ln \mathbb{G})}}=\frac{\sum_{i=1}^{n}\left(Z_{i}-\mathrm{E}\left[Z_{i}\right]\right)}{\sqrt{\operatorname{Var}\left(\sum_{i=1}^{n} Z_{i}\right)}} .
$$

Using the fact that the geometric average is not greater than the arithmetic average, a possible decomposition variable so that $\Lambda \geq d_{\Lambda}$ implies that $\mathbb{S} \geq d$ is in this case given by

$$
d_{\Lambda}=\frac{n \ln \left(\frac{d}{n}\right)-\sum_{i=1}^{n} \mathrm{E}\left[Z_{i}\right]}{\sqrt{\operatorname{Var}\left(\sum_{i=1}^{n} Z_{i}\right)}}
$$

Note that the aforementioned decomposition variables $d_{\Lambda}$ depend on the retention $d$ and that when the retention $d$ tends to infinity also these $d_{\Lambda}$ do. The error bound $\varepsilon\left(d_{\Lambda}\right)(24)$ will then converge to a constant so that the upper bound $\pi^{d e u b}(\mathbb{S}, d, \Lambda)$ will not converge to zero but to this constant instead. The exact part in the partially exact/comonotonic upper bound $\pi^{\text {pecub }}(\mathbb{S}, d, \Lambda)$ will become zero and hence the upper bound itself will coincide with the improved comonotonic upper bound $\pi^{i c u b}(\mathbb{S}, d, \Lambda)$ which will tend to zero. This explains the behaviour that for large retentions $d$ the upper bounds $\pi^{\text {pecub }}(\mathbb{S}, d, \Lambda)$ and $\pi^{i c u b}(\mathbb{S}, d, \Lambda)$ will outperform the upper bound $\pi^{d e u b}(\mathbb{S}, d, \Lambda)$.

The three choices above for $\Lambda$ will not lead to a good performance for the improved comonotonic upper bound since those choices will not only cause the first order approximation of the variance of $\mathbb{S}^{\ell}$ to be large but also the first order approximation of the variance of $\mathbb{S}^{u}$ while we just want the opposite for the latter variance. To determine a good choice for $\Lambda$ we should make the correlation structure of the components in $\mathbb{S}^{u}$ as close as possible to the correlation structure of those in $\mathbb{S}$. This is attained by putting $\Lambda$ equal to a $Z_{k}$ for some $k \in\{1, \ldots, n\}$ such that the difference

$$
\left|r_{i} r_{j}+\sqrt{1-r_{i}^{2}} \sqrt{1-r_{j}^{2}}-\operatorname{Corr}\left(Z_{i}, Z_{j}\right)\right|
$$

is small for most of the $i$ and $j$, where $r_{i}$ is defined in (10).

In the remainder of this paper, the choice of $\Lambda$ will be dependent on the time horizon $n$. To indicate this dependence, we introduce the notation $\Lambda_{n}$ for the used conditioning variable $\Lambda$.

\section{Bounds for compound sums}

Bounds for the right tails (4) of a compound sum $\mathbb{S}_{N}(3)$ are obtained by bounding the different right tails composing the sum (4). It is straightforward to obtain a lower bound, 
denoted as $\pi^{\ell b}\left(\mathbb{S}_{N}, d, \boldsymbol{\Lambda}\right)$, by looking at the combination

$$
\pi^{\ell b}\left(\mathbb{S}_{N}, d, \boldsymbol{\Lambda}\right)=\sum_{j=1}^{\infty} \operatorname{Pr}(N=j) \pi^{\ell b}\left(\mathbb{S}_{j}, d, \Lambda_{j}\right)
$$

with $\boldsymbol{\Lambda}=\Lambda_{1}, \Lambda_{2}, \ldots$ and $\pi^{\ell b}\left(\mathbb{S}_{j}, d, \Lambda_{j}\right)$ given by (12) for $n=j$. The same reasoning can be followed for obtaining the comonotonic upper bound $\pi^{c u b}\left(\mathbb{S}_{N}, d\right)$, the improved comonotonic upper bound $\pi^{i c u b}\left(\mathbb{S}_{N}, d, \boldsymbol{\Lambda}\right)$ and the partially exact/comonotonic upper bound $\pi^{\text {pecub }}\left(\mathbb{S}_{N}, d, \boldsymbol{\Lambda}\right)$.

For each term $\pi\left(\mathbb{S}_{j}, d\right)$ in the sum (4) we can take the minimum of two or more of the above defined upper bounds. We propose two upper bounds based on this simple idea.

The first bound takes each time the minimum of the error term (17) independent of the retention and the error term (22) dependent on the retention (although in practical applications one notices that the minimum is obtained by the error term (22)). Combining this with the stop-loss premium of the lower bound $\mathbb{S}^{\ell}$ results in the following upper bound

$$
\pi^{e m u b}\left(\mathbb{S}_{N}, d, \boldsymbol{\Lambda}\right)=\pi^{\ell b}\left(\mathbb{S}_{N}, d, \boldsymbol{\Lambda}\right)+\sum_{j=1}^{\infty} \operatorname{Pr}(N=j) \min \left(\frac{1}{2} \mathrm{E}\left[\sqrt{\operatorname{Var}\left[\mathbb{S}_{j} \mid \Lambda_{j}\right]}\right], \varepsilon\left(d_{\Lambda_{j}}\right)\right) .
$$

Calculating for each term the minimum of all presented upper bounds

$$
\begin{aligned}
& \pi^{\min }\left(\mathbb{S}_{N}, d, \boldsymbol{\Lambda}\right) \\
& =\sum_{j=1}^{\infty} \operatorname{Pr}(N=j) \min \left(\pi^{c u b}\left(\mathbb{S}_{j}, d\right), \pi^{i c u b}\left(\mathbb{S}_{j}, d, \Lambda_{j}\right), \pi^{\text {pecub }}\left(\mathbb{S}_{j}, d, \Lambda_{j}\right), \pi^{\text {emub }}\left(\mathbb{S}_{j}, d, \Lambda_{j}\right)\right)
\end{aligned}
$$

will of course provide a better upper bound, which can even be improved by using different $\Lambda_{j}$ for deriving the different upper bounds for each term $\pi\left(\mathbb{S}_{j}, d\right)$ in the sum (4).

Remark that $\pi^{e m u b}\left(\mathbb{S}_{j}, d, \Lambda_{j}\right)=\pi^{\ell b}\left(\mathbb{S}_{j}, d, \Lambda_{j}\right)+\min \left(\frac{1}{2} \mathrm{E}\left[\sqrt{\operatorname{Var}\left[\mathbb{S}_{j} \mid \Lambda_{j}\right]}\right], \varepsilon\left(d_{\Lambda_{j}}\right)\right)$.

\section{$6 \quad$ Numerical illustrations}

In this section, we apply the different lower and upper bounds presented in the previous sections to the life annuity problems discussed in Section 2. We compare the performance of these bounds in a numerical illustration.

Recall that $\tilde{\mathbb{S}}_{j}$ in $(5)$ and $\mathbb{S}^{\text {average }}$ in $(6)$ are both of the form $(7)$ with $Z_{i}=Y(i)=$ $Y_{1}+\cdots+Y_{i}$. In the numerical illustrations, we assume that these yearly returns $Y_{i}$ are i.i.d. normally distributed with mean $\mu=0.07$ and volatility $\sigma=0.1$.

In order to compute the lower and upper bounds for the stop-loss premiums, we consider as conditioning r.v. $\Lambda_{n}=\sum_{i=1}^{n} \gamma_{i} Z_{i}$ with $\gamma_{i}$ given by (27) in the 'Taylor-based' case and $\gamma_{i}$ given by (29) in the 'maximal variance' case, and with corresponding decomposition 
variable of the form (28). For the numerical illustrations in this section we present each time the one which provides the best result.

Remark that the correlation coefficients $r_{i}$ are given by

$$
r_{i}=\frac{\sum_{j=1}^{i} \sum_{k=j}^{n} \gamma_{k}}{\sqrt{i \sum_{j=1}^{n}\left(\sum_{k=j}^{n} \gamma_{k}\right)^{2}}} .
$$

As they are positive, the formulas (14) and (15) can be applied.

The 'maximal variance' conditioning variable (29) seems to perform better far in the tail. So for high values of $d$ the different bounds based on this conditioning variable approximate more accurate the real value of the right tails than the approximations using the 'Taylorbased' conditioning variable (27). In our numerical illustrations, the 'geometric average' conditioning variable performs in general slightly worse than the two others.

Hereafter, we compare the performance of the following bounds presented in sections 3 and 4: the lower bound $\pi^{\ell b}(\mathbb{S}, d, \Lambda)$ (LB), the comonotonic upper bound $\pi^{c u b}(\mathbb{S}, d)$ (CUB), the improved comonotonic upper bound $\pi^{i c u b}(\mathbb{S}, d, \Lambda)$ (ICUB), the upper bound based on the lower bound $\pi^{e u b}(\mathbb{S}, d, \Lambda)(\mathrm{EUB})$ and $\pi^{\text {deub }}(\mathbb{S}, d, \Lambda)$ (DEUB) and the partially exact/comonotonic upper bound $\pi^{\text {pecub }}(\mathbb{S}, d, \Lambda)$ (PECUB). For applications with a stochastic time horizon $N$ we also consider the bounds from section 5 with in particular the two combination bounds $\pi^{e m u b}\left(\mathbb{S}_{N}, d, \boldsymbol{\Lambda}\right)(\mathrm{EMUB})$ and $\pi^{\min }\left(\mathbb{S}_{N}, d, \boldsymbol{\Lambda}\right)(\mathrm{MIN})$.

In addition, we calculate the stop-loss premiums by Monte-Carlo simulation (MC). These MC estimates serve as a benchmark for the different lower and upper bounds. The simulation results are based on generating $50 \times 1000000$ paths and antithetic variables are used to reduce the variance of the Monte-Carlo estimates. For each estimate we computed the standard error (s.e.). As is well-known, the (asymptotic) 95\% confidence interval is given by the estimate plus or minus 1.96 times the standard error. Such a simulation is time-consuming compared to the computation of the analytical expressions for the bounds.

For the life annuity with present value $\mathbb{S}^{\text {policy }}(2)$, we consider a male person of age 65 . The different lower and upper bounds for the right tails of the present value of a whole life annuity due of 1 payable at the end of each survival year (annuity-immediate) are compared in Table 1. For the retentions $d=5,10$ and 15 the upper bound MIN improves the comonotonic and improved comonotonic upper bounds CUB and ICUB, respectively. For larger values of $d$, all approximations are more or less the same. Up to a retention $d=15$ the upper bound EMUB performs better than the ICUB and the PECUB bounds, but from $d=20$ on it is the other way around as was predicted earlier when discussing the limiting behaviour of these bounds. Overall, the lower bound LB derived from the methodology proposed in Kaas et al. (2000) seems to be the closest approximation for the right tails or stop-loss premiums.

Table 2 shows the results for the right tails $\mathrm{E}\left[\left(\mathbb{S}^{\text {average }}-d\right)_{+}\right]$for different values of $d$. Again the lower bound approach as proposed in Kaas et al. (2000) approximates the 


\begin{tabular}{|l|l|l|l|l|l|l|l|}
\hline & $d=0$ & $d=5$ & $d=10$ & $d=15$ & $d=20$ & $d=25$ & $d=30$ \\
\hline LB & 9.3196 & 4.6191 & 1.2269 & 0.1737 & 0.0207 & 0.0026 & 0.0004 \\
MC & 9.3196 & 4.6191 & 1.2304 & 0.1739 & 0.0216 & 0.0026 & 0.0004 \\
$\left(\right.$ s.e. $\left.\times 10^{5}\right)$ & & $(8.49)$ & $(5.48)$ & $(0.51)$ & $(0.19)$ & $(0.01)$ & $(0.002)$ \\
ICUB & 9.3196 & 4.6238 & 1.3277 & 0.2530 & 0.0454 & 0.0088 & 0.0019 \\
CUB & 9.3196 & 4.6244 & 1.3389 & 0.2610 & 0.0480 & 0.0095 & 0.0021 \\
EMUB & 9.3196 & 4.6197 & 1.2400 & 0.2145 & 0.0718 & 0.0545 & 0.0522 \\
PECUB & 9.3196 & 4.6219 & 1.2839 & 0.2381 & 0.0451 & 0.0088 & 0.0019 \\
MIN & 9.3196 & 4.6195 & 1.2385 & 0.2070 & 0.0444 & 0.0088 & 0.0019 \\
\hline
\end{tabular}

Table 1: Approximations for right tails at level $d$ of $\mathbb{S}^{\text {policy }}$.

exact stop-loss premiums extremely well. The results for the upper bounds are in line with the previous ones. Note that for very high values of $d$ the differences become larger.

\begin{tabular}{|l|l|l|l|l|}
\hline & $d=0$ & $d=5$ & $d=10$ & $d=15$ \\
\hline LB & 9.3196 & 4.3200 & 0.5533 & 0.0193 \\
MC & 9.3196 & 4.3200 & 0.5543 & 0.0197 \\
$\left(\right.$ s.e. $\left.\times 10^{5}\right)$ & & $(0.37)$ & $(0.13)$ & $(0.035)$ \\
ICUB & 9.3196 & 4.3227 & 0.7076 & 0.0523 \\
CUB & 9.3196 & 4.3233 & 0.7217 & 0.0559 \\
EUB & 9.3751 & 4.3755 & 0.6090 & 0.0749 \\
DEUB & 9.3196 & 4.3202 & 0.5784 & 0.0744 \\
PECUB & 9.3196 & 4.3219 & 0.6515 & 0.0522 \\
\hline
\end{tabular}

Table 2: Approximations for right tails with retention $d$ of $\mathbb{S}_{x}^{\text {average }}$.

Remark that only for EUB the error term is independent of the retention and therefore in both tables all values for $d=0$, except these for EUB, are identical and equal to 9.3196. This follows from the fact that in this case the expected value of $\mathbb{S}^{\text {policy }}$ equals the expected value of $\mathbb{S}^{\text {average }}$. Note also that the values in Table 1 are typically larger than the corresponding values in Table 2. This is not surprising. From Example 1 in Hoedemakers et al. (2004) it immediately follows that $\mathbb{S}^{\text {average }} \leq_{c x} \mathbb{S}^{\text {policy }}$ and hence for any $d>0$ one has

$$
\mathrm{E}\left[\left(\mathbb{S}^{\text {average }}-d\right)_{+}\right] \leq \mathrm{E}\left[\left(\mathbb{S}^{\text {policy }}-d\right)_{+}\right]
$$

\section{$7 \quad$ Summary and conclusions}

In this paper we consider several methodologies for approximating right tails of nonindependent r.v.'s. We compare and generalize methods proposed by Curran (1994), 
Rogers and Shi (1995), Kaas et al. (2000), Dhaene et al. (2002a,b), Nielsen and Sandmann (2003) and Vanmaele et al. (2006). In particular, we consider bounds based on comonotonicity as proposed in Kaas et al. (2000). Further, we concentrate upon the upper bound obtained by adding an error term to the lower bound of Rogers and Shi (1995). We explain how these bounds can be improved by decomposing an integral formula for the right tails into two parts: one can be easily solved analytically, the other part can be approximated by one of the comonotonic upper bounds. We generalize several existing bounds to the case of compound sums.

All the presented approximations are applied to the stochastic present value of the liabilities associated with a well-diversified average portfolio of life annuities as well as with a single life annuity. In the latter case it is possible to decompose the value of the stop-loss premium by conditioning and apply the best (smallest) upper bound on each of the components separately. We provide a number of numerical illustrations which show that the decomposition significantly improves the bounds.

\section{Acknowledgments}

Jan Dhaene acknowledges the financial support of the Onderzoeksfonds K.U.Leuven (GOA/02: Actuariële, financiële en statistische aspecten van afhankelijkheden in verzekerings- en financiële portefeuilles).

Michèle Vanmaele would like to acknowledge the financial support by the BOF-project 001104599 of the Ghent University.

Griselda Deelstra acknowledges financial support of the BNB-contract FC0315D00000.

\section{References}

[1] Bowers, N.L., Gerber, H.U., Hickman, J.C., Jones, D.A. and Nesbitt, C.J. (1986). Actuarial mathematics. Schaumburg, Ill.: Society of Actuaries.

[2] Curran, M. (1994). Valuing Asian and portfolio options by conditioning on the geometric mean price. Management Science, 40(12), 1705-1711.

[3] Deelstra, G., Diallo, I. and Vanmaele, M. (2008). Bounds for Asian basket options, Journal of Computational and Applied Mathematics, 218, 215-228.

[4] Deelstra, G., Liinev, J. and Vanmaele, M. (2004). Pricing of arithmetic basket options by conditioning, Insurance: Mathematics and Economics, 34(1), 55-77.

[5] Denneberg, D. (1994). Non-Additive Measure and Integral. Theory and Decision Library, Series B, 27, Kluwer Academic Publishers.

[6] Denuit, M., Dhaene, J., Goovaerts, M.J. and Kaas, R. (2005). Actuarial Theory for Dependent Risk: Measures, Orders and Models. Wiley, New York. 
[7] De Vylder, F. and Goovaerts, M.J. (1982). Upper and lower bounds on stop-loss premiums in case of known expectation and variance of the risk variable. Mitt. Verein. Schweiz. Versicherungmath., 149-164.

[8] Dhaene, J., Denuit, M., Goovaerts, M.J., Kaas, R. and Vyncke, D. (2002a). The concept of comonotonicity in actuarial science and finance: theory. Insurance: Mathematics and Economics, 31(1), 3-33.

[9] Dhaene, J., Denuit, M., Goovaerts, M.J., Kaas, R. and Vyncke, D. (2002b). The concept of comonotonicity in actuarial science and finance: applications. Insurance: Mathematics and Economics, 31(2), 133-161.

[10] Dhaene, J., Vanduffel, S., Goovaerts, M.J., Kaas, R., Vyncke, D. (2005). Comonotonic approximations for optimal portfolio selection problems. Journal of Risk and Insurance, 72 (2), 253-301.

[11] Hoedemakers, T., Darkiewicz, G., Dhaene, J. and Goovaerts, M.J. (2004). On the Distribution of Life Annuities with Stochastic Interest Rates. Proceedings of the Eighth International Congress on Insurance: Mathematics and Economics, Rome.

[12] Hürlimann, W. (1996). Improved analytical bounds for some risk quantities. ASTIN Bulletin, 26(2), 185-199.

[13] Hürlimann, W. (1998). On best stop-loss bounds for bivariate sums by known marginal means, variances and correlation. Mitt. Verein. Schweiz. Versicherungmath., 111-134.

[14] Hürlimann, W. (2002). Analytical bounds for two Value-at-Risk functionals. ASTIN Bulletin, 32, 235-265.

[15] Jansen, K., Haezendonck, J. and Goovaerts, M.J. (1986). Upper bounds on stop-loss premiums in case of known moments up to the fourth order. Insurance: Mathematics and Economics, 5(4), 315-334.

[16] Kaas, R., Dhaene, J. and Goovaerts, M.J. (2000). Upper and lower bounds for sums of random variables. Insurance: Mathematics and Economics, 27(2), 151-168.

[17] Laeven, R.J.A., Goovaerts, M.J. and Hoedemakers, T. (2005). Some asymptotic results for sums of dependent random variables with actuarial applications. Insurance: Mathematics and Economics, 37(2), 154-172.

[18] Landsberger M. and Meilijson, I. (1994). Co-monotone allocations, Bickel-Lehman dispersion and the Aroow-Pratt measure of risk aversion. Annals of Operations Research, 52, 97-106.

[19] Nielsen, J.A. and Sandmann, K. (2003). Pricing bounds on Asian options. Journal of Financial and Quantitative Analysis, 38(2), 449-473. 
[20] Reynaerts, H., Vanmaele, M., Dhaene, J. and Deelstra, G. (2006). "Bounds for the price of a European-style Asian option in a binary tree model", European Journal of Operational Research, 168(2), 322-332.

[21] Rogers, L.C.G. and Shi, Z. (1995). The value of an Asian option. Journal of Applied Probability, 32, 1077-1088.

[22] Simon, S., Goovaerts, M.J. and Dhaene, J. (2000). An easy computable upper bound for the price of an arithmetic Asian option. Insurance: Mathematics and Economics, 26(2-3), 175-184.

[23] Vanduffel, S., Hoedemakers, T. and Dhaene, J. (2005). Comparing approximations for risk measures of sums of non-independent lognormal random variables. North American Actuarial Journal, 9(4), 71-82.

[24] Vanmaele, M., Deelstra, G. and Liinev, J. (2004). Approximation of stop-loss premiums involving sums of lognormals by conditioning on two random variables. Insurance: Mathematics and Economics, 35(2), 343-367.

[25] Vanmaele, M., Deelstra, G., Liinev, J., Dhaene, J. and Goovaerts M.J. (2006). Bounds for the price of discrete arithmetic Asian options. Journal of Computational and Applied Mathematics, 185(1), 51-90. 